\title{
Langerhans cells renew in the skin throughout life under steady-state conditions
}

Miriam Merad, Markus G. Manz, Holger Karsunky, Amy Wagers, Wendy Peters, Israel Charo, Irving L.Weissman, Jason G. Cyster and Edgar G. Engleman

\section{Nature Immunology 3, 1135-1141}

In the December 2002 issue of Nature Immunology, incorrect versions of Figures $1 \mathrm{f}$ and $4 \mathrm{~g}$ were supplied. The correct versions of these fig-

ures are shown below. This mistake does not affect the message of the paper.

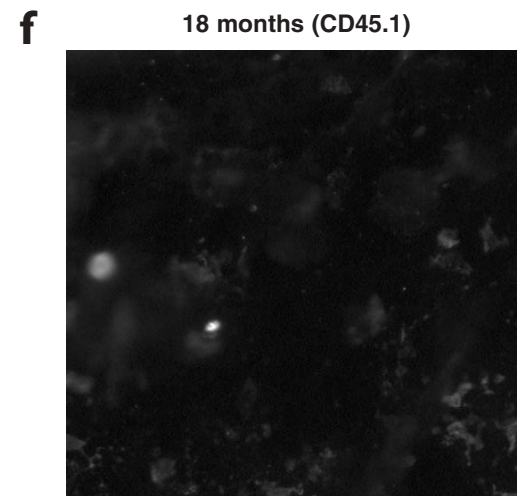

Figure I. LC chimerism after congenic BM transplantation. Eight-week-old CD45.2-expressing C57BL/6 mice were lethally irradiated and injected intravenously with $5 \times 10^{5}$ BM cells isolated from congenic CD45. I-expressing mice. (a) Contour plots show the expression of CD45.I and CD45.2 on gated I-A $\mathrm{A}^{\mathrm{b}+} \mathrm{CD}$ I Ic $\mathrm{c}^{+}$spleen and kidney DCs, CDII lb+Gr-I-B220-NKI.I- peritoneal macrophages and $\mathrm{CDIIb}^{+} \mathrm{Gr}-\mathrm{I}^{-}$ blood monocytes isolated 8 weeks after transplantation. The percentages of CD45. $\mathrm{I}^{+}$ and CD45.2 -gated cells are indicated. (b,c) Epidermal cells were isolated before and at different times after congenic BM transplantation; three to six mice were analyzed independently for each time point. (b) One representative experiment showing the expression of CD45.I and CD45.2 on gated I-A $\mathrm{A}^{b+} \mathrm{CD} / \mathrm{Ic}^{+}$epidermal LCs. (c) Bar graphs show the mean percentages of LCs that were donor (CD45.I)- or host (CD45.2)-derived at different times after transplantation. (d-f) Epidermal sheets were obtained from (d) nonreconstituted (naïve) C57BL/6 mice or (e,f) chimeric animals 18 months after congenic BM transplantation and stained with (d,e) CD45.2 or (f) CD45.I mAbs. (g) DC chimerism in inguinal (skin-draining), mesenteric and spleen LNs was determined at 6 months after congenic BM transplantation in four different mice. Bar graphs show the percentages of $\mathrm{I}-\mathrm{A}^{\mathrm{b}+} \mathrm{CD} / \mathrm{lc} \mathrm{c}^{+} \mathrm{DCs}$ that expressed $\mathrm{CD} 45.2$ in different secondary lymphoid organs in each reconstituted animal.

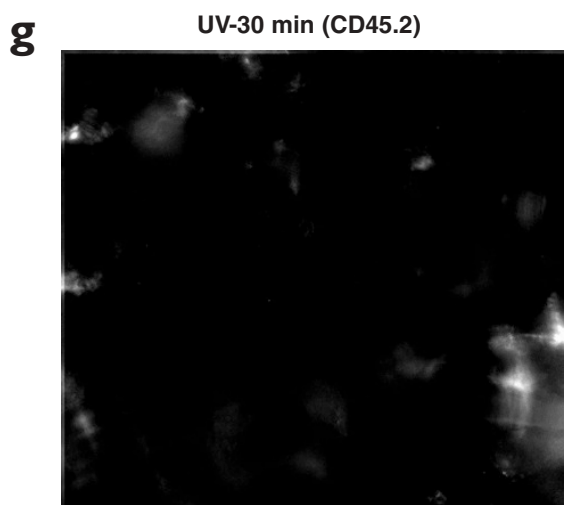

Figure 4. LC homeostasis during skin inflammation. CD45.2 $2^{+}$mice were lethally irradiated and reconstituted with $5 \times 10^{5} \mathrm{CD} 45.1^{+} \mathrm{BM}$ cells and either left untreated or exposed 8 weeks later to UV light for 10 or $30 \mathrm{~min}$ or to a mixture of oil and acetone applied to each ear. $(\mathbf{a}, \mathbf{b})$ Three ears from three different animals were collected at different times after skin injury and analyzed to determine (a) the total number of LCs per ear and (b) the percentage of epidermal cells that were host LCs. (c) Dot plots show the expression of CD45.I and CD45.2 on

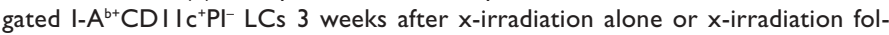
lowed by skin irritation or UV exposure. (d) Bar graphs show the mean percentages of total epidermal cells that were host- or donor-derived LCs analyzed 3 weeks after skin injury in three different animals. (e-g) Images of epidermal sheets obtained from C57BL/6 animals reconstituted with CD45. I $^{+}$BM 12 weeks after exposure to UV for $30 \mathrm{~min}$ and stained with (e) CD45.I, (f) I-A $\mathrm{A}^{\mathrm{b}}$ or (g) CD45.2 mAbs. Magnification: $\times 20$. 